\title{
Value of beat-to-beat blood pressure changes, detected by pulse transit time, in the management of the obstructive sleep apnoea/ hypopnoea syndrome
}

\author{
D.J. Pitson, J.R. Stradling
}

Value of beat-to-beat blood pressure changes, detected by pulse transit time, in the management of the obstructive sleep apnoea/hypopnoea syndrome. D.J. Pitson, J.R. Stradling. CERS Journals Ltd 1998.

ABSTRACT: Two important aspects of a respiratory sleep study are a measure of inspiratory effort and an estimate of the number of arousals. These can be derived from an indirect estimate of beat-to-beat blood pressure (BP), pulse transit time (PTT). This study investigated the reproducibility of inspiratory BP falls (reflecting inspiratory effort), and BP arousals derived from PTT, and the contribution they could make to the management of the obstructive sleep apnoea/hypopnoea syndrome (OSAHS).

Overnight PTT was recorded at home in $\mathbf{4 0}$ patients being investigated for OSAHS, and a second PTT recording was made in the sleep laboratory with full polysomnography. Patients were divided into three groups according to the severity of their sleep disorder, and a third PTT recording was made at home in 13 patients subsequently established on nasal continuous positive airway pressure (CPAP).

The reproducibility between the home and laboratory studies was reasonable $(r=0.87$ for inspiratory BP falls, $r=0.81$ for BP arousals). Both derivatives showed a clear progression through the three patient groups, which returned to normal on treatment. The differences between the groups were significant $(p<0.001$ for inspiratory BP falls, $p=0.0014$ for $B P$ arousals). Receiver operator characteristic curves, used to compare polysomnography variables and PTT variables, confirmed that the PTT variables were as good as apnoea-hypopnoea index (AHI), $>4 \%$ arterial oxygen saturation dip rate and electroencephalography micro-arousals at dividing patients into two groups, either requiring nasal CPAP or not requiring CPAP.

Pulse transit time can provide a noninvasive estimate of inspiratory effort and a measure of arousals that together document disease severity and response to treatment and may be useful in managing obstructive sleep apnoea/hypopnoea syndrome. Eur Respir J 1998; 12: 685-692.

Osler Chest Unit, The Churchill, Oxford Radcliffe Hospital, Oxford, UK.

Correspondence: D.J. Pitson

Osler Chest Unit

The Churchill

Oxford Radcliffe Hospital

Oxford OX3 7LJ

UK

Fax: 441865225221

Keywords: Obstructive sleep apnoea pulse transit time

Received: December 21997

Accepted after revision April 271998

This work was supported by a grant from the British Lung Foundation.
Daytime hypersomnolence is one of the classical features of the obstructive sleep apnoea/hypopnoea syndrome (OSAHS) [1] and the symptom for which treatment is usually prescribed [2]. Daytime sleepiness results from the many transient arousals that fragment sleep in OSAHS and that are believed to be a response to the increases in inspiratory effort being made in an attempt to maintain adequate ventilation through a narrowed or collapsed upper airway $[3,4]$.

A respiratory sleep study to investigate OSAHS should therefore include either a measure of ventilatory effort or upper airway narrowing, and a measure of arousals as a marker of the resulting sleep fragmentation [5]. Because OSAHS is a relatively common disorder affecting 1-5\% of the adult male population [6], and the financial resources for healthcare are limited, such respiratory sleep studies should also be cost-effective and, preferably, domiciliary [7].

The arousal process that occurs in response to alerting stimuli begins at the brain stem level with a reflex increase in sympathetic activity causing changes in a number of measurable variables for example, blood pressure, heart rate and skin vasoconstriction [8]. For minor alerting stimuli, this may be the only level of response although, for more significant stimuli, the arousal process will radiate upwards to involve the cortex causing changes in the electroencephalogram (EEG) and, eventually, full awakening from sleep. There is some debate as to what level of activation of the nervous system constitutes an "arousal", but for the purposes of this paper, the term arousal is taken as a general term to reflect activation of the nervous system at any level in response to an alerting stimulus. Autonomic responses to alerting stimuli are termed "autonomic arousals" and, more specifically, the blood pressure (BP) rises measured in this study are termed "BP arousals". Arousal responses involving cortical activation are termed "cortical arousals", and the brief EEG changes measured in this study are termed "EEG micro-arousals". The exact relationship between the recurrent arousals and the consequent daytime sleepiness has also not yet been established, but there is evidence to suggest that arousals that are detectable by autonomic changes, but where there is no EEG 
change (i.e. no cortical arousal), may result in daytime sleepiness $[9,10]$. Thus, measuring autonomic arousals may be as important as measuring cortical arousals.

Systolic blood pressure (SBP) falls during inspiration (pulsus paradoxus), and the size of this inspiratory fall is proportional to the degree of inspiratory effort [11]. There are also rises in SBP with each arousal from sleep that occur even in response to extremely minor alerting stimuli that produce no discernible change in the EEG [12]. DAVIES et al. [13] recorded beat-to-beat BP using the Finapres device and developed computer algorithms to identify inspiratory SBP falls and arousal-related rises in BP from the all night data. These algorithms were subsequently used to identify patients with sleep related breathing disorders.

The Finapres technology used by DAVIES et al. [13] has an inflating cuff fitting around the finger. This is uncomfortable if worn for long periods and the control unit is large; therefore it is less than ideal for routine overnight monitoring, and is unsuitable for domiciliary sleep studies. Because of these limitations, we have been investigating an indirect estimate of beat-to-beat BP, pulse transit time (PTT) [14], which can be recorded and stored by a portable device.

PTT is the transmission time of the arterial pulse pressure wave from the aortic valve to the periphery and is usually recorded as the time between the R-wave on the electrocardiogram (ECG) and the subsequent arrival of the corresponding pulse shock wave at the finger. This is typically around $250 \mathrm{~ms}$ and is thus much faster than the time taken for the blood ejected by the left ventricle to actually reach the periphery (usually about $25 \mathrm{~s}$ ). The principal determinant of the speed with which the shock wave travels is the degree of stiffness/tension in the ar-terial walls that in turn is determined mostly by the BP. Thus, as BP increases, there is an increase in the stiffness/tension of the arterial walls and the pulse wave travels faster (PTT decreases). Conversely, as BP falls, there is less stiffness/ tension in the arterial walls, and the pulse wave travels more slowly (PTT increases).

We have shown that the inspiratory BP falls can be detected by increases in PTT $[15,16]$ and that the size of these increases are related to the degree of inspiratory effort [17]. In addition, the BP rise with arousal can be detected by a decrease in PTT [18], and the number of BP rises per hour correlate well with other indices of sleep fragmentation [19]. We have adapted the algorithms of DAVIES et al. [13] to detect inspiratory BP falls (estimate of inspiratory effort) and arousal-related rises in BP (estimate of sleep fragmentation) from an all-night PTT recording.

The aim of this study was to provide some evidence regarding the reproducibility of these two derivatives of the PTT recording and to examine the possible contribution that they could make in managing the OSAHS.

\section{Materials and methods}

\section{Study subjects}

Forty patients who were referred to the Oxford Sleep Unit for investigation of possible obstructive sleep apnoea were recruited to the study. Thirty-four were male, and six were female. Their mean age was 48 yrs (range 23-66 yrs), and their mean body mass index was 31 (range 24-50).
The study was approved by the Central Oxford Research Ethics Committee (project number 2792).

\section{Methods}

Pulse transit time. The PTT for each heart beat was calculated, recorded and stored by an RM10 recorder (Parametric Recorders, London, UK). Three thoracic electrodes were used to sample the ECG, and the arrival of the pulse shock wave at the finger was detected photometrically by an oximeter finger probe. PTT is measured in real time as the interval from the QRS complex to a threshold on the rising edge of the finger pulse. The threshold is calculated as $25 \%$ of the height of the pulse during a time window of $280 \mathrm{~ms}$ after the QRS. The QRS is detected by sampling the ECG at $500 \mathrm{~Hz}$ and the pulse is also sampled at 500 $\mathrm{Hz}$, giving an accuracy of $2 \mathrm{~ms}$. A new value for PTT is only available with each heart beat, and therefore, to ensure that none is missed, PTT values are sampled at $5 \mathrm{~Hz}$ and these are the values stored in the RM10.

Polysomnography. One channel of EEG (either C3/A2 or C4/A1), separate electro-oculograms (EOGs) for each eye, and submental electromyograms (EMG) were recorded. Oronasal airflow was measured using a thermistor; ribcage and abdominal movements were measured by inductance plethysmography from belts around the chest and abdomen. These signals were recorded using an MPA-2 recorder (Oxford Medical, Oxford, UK).

Arterial oxygen saturation $\left(\mathrm{Sa}, \mathrm{O}_{2}\right)$ and pulse rate were measured by an Ohmeda 3700 pulse oximeter (Ohmeda, Boulder, CA, USA) and were recorded along with body movements and snoring by the Visi-Lab sleep monitoring system (Stowood Scientific Instruments, Oxford, UK).

Clinical diagnosis. Subjects were classified into three groups according to their clinical diagnosis. Routine management of these patients involves an outpatient assessment history, Epworth Sleepiness Score (ESS) [20], examination, and a Visi-Lab sleep study. The sleep study is reviewed to assess obstructive respiratory problems (hypoxic dips and snoring) and sleep disturbance (body movements and heart rate oscillations). The clinical diagnosis is then made, based on the integration of history, sleepiness and sleep study findings.

The three clinical groups, reflecting their subsequent management, were: 1) normal, virtually no snoring and no sleep disturbance due to OSAHS; 2) mild/moderate, snoring, limited evidence of sleep disruption and/or insufficient daytime sleepiness for the clinician and patient to feel a trial of nasal CPAP was justified; and 3) severe disorder, evidence of upper airway obstruction (snoring, hypopnoeas, apnoeas, hypoxic dips) and daytime symptoms (usually sleepiness) to justify a trial of nasal continuous positive airway pressure (CPAP).

This clinical allocation into management groups is used in our unit because of the failure of any particular sleep study derivative (e.g. apnoea-hypopnoea index (AHI)) to accurately identify those patients likely to benefit from nasal CPAP [2]. Table 1 shows the median and upper/lower quartiles of four severity indicators for the three groups, both those available at the time of diagnosis (ESS, $>4 \%$ $\mathrm{Sa}_{\mathrm{a}, \mathrm{O}_{2}}$ dip rate), and those used only for this study (AHI, EEG micro-arousals $\left.\cdot h^{-1}\right)$. 
Table 1. - Clinical characteristics of patients grouped according to the severity of their sleep disorder

\begin{tabular}{lcccc}
\hline Clinical group & $\begin{array}{c}\text { ESS } \\
\text { median }(25-75 \%)\end{array}$ & $\begin{array}{c}\mathrm{Sa}_{\mathrm{a}} \mathrm{O}_{2} \text { dips.h-1 } \\
\text { median }(25-75 \%)\end{array}$ & $\begin{array}{c}\text { AHI } \\
\text { median }(25-75 \%)\end{array}$ & $\begin{array}{c}\text { EEG micro-arousals.h-1 } \\
\text { median }(25-75 \%)\end{array}$ \\
\hline Normal $(\mathrm{n}=11)$ & $10(7-15)$ & $1.3(0.9-2.5)$ & $4.5(2.3-9.5)$ & $19.0(12.0-31.0)$ \\
Mild/moderate $(\mathrm{n}=11)$ & $9(5-13)$ & $2.5(1.0-4.5)$ & $8.5(3.3-10.5)$ & $20.0(14.0-28.0)$ \\
Severe disorder $(\mathrm{n}=18)$ & $16(11-18)$ & $17.9(4.3-46.5)$ & $32.0(22.0-53.0)$ & $37.5(24.5-61.3)$ \\
\hline
\end{tabular}

ESS: Epworth Sleepiness Score; AHI: apnoea/hypopnoea index; $\mathrm{Sa}_{\mathrm{a}} \mathrm{O}_{2}$ : arterial oxygen saturation; EEG: electroencephalogram.

\section{Protocol}

The study was conducted in three stages. Initially, a recording of overnight PTT was undertaken in the patient's own home. On a separate occasion, the patient was admitted to the sleep unit where a second overnight PTT recording was made in conjunction with a full research polysomnography sleep study and Visi-lab study. At this stage, patients were also grouped according to the severity of their sleep disorder into management groups as above, without reference to the polysomnography results. Finally, those patients who were commenced on nasal CPAP were restudied at home with a further PTT recording enabling us to examine the change in the PTT derivatives after treatment.

Domiciliary recording. The home visits were undertaken by one of the authors (D.J. Pitson) who set up the RM10 recorder using a lap-top PC (Commodore 286-LT, West Chester, PA, USA). The three thoracic electrodes (Unilect $\mathrm{Ag} / \mathrm{AgCI}, \mathrm{MSB}$, Wiltshire, UK) were positioned to ensure a clear positive $\mathrm{R}$ wave. The finger probe was attached to the index finger of the right hand. The RM10 was programmed to start recording approximately $1 \mathrm{~h}$ before the patient's usual bed time and stop recording about an hour after their usual wake time. Once adequate signals had been verified, the PC was disconnected from the RM10, and the RM10 was disconnected from the patient, leaving just the three ECG electrodes in place. The patient was instructed to reconnect the ECG leads and finger probe when they went to bed and to tape over the leads with sticky tape to try and prevent accidental disconnection during the study. An instruction sheet reminding the patient of what they needed to do was left together with a form asking them to record how well they slept on a threepoint scale: same as normal; a little less than normal; or much less than normal.

Polysomnography. Patients were asked to attend for their hospital sleep study at 20:00 h. All the recording equipment was attached, and the MPA-2, RM10 and Visi-lab sleep monitoring system were time-synchronized. Patients were allowed to remove the monitoring equipment themselves when they woke up in the morning and completed the same questionnaire to record how well they slept, as in the home study.

Repeat domiciliary recording. For those patients in the severe group who were successfully established on nasal CPAP, a second home RM10 study was performed at least 3 months after CPAP treatment had begun. This was identical to the first home study.

\section{Analysis}

PTT data. The all-night PTT recording was viewed, and any artefactual periods at the start of the recording (pre- sumed to be while the patient was attaching to the equipment) and at the end of the recording (presumed to be after the patient had woken) were excluded. The intervening period was automatically processed by the RM50 software version 1.51 (Parametric Recorders, London, UK) for BP arousals and inspiratory BP falls. If any of the equipment became detached during the night, the available time period was analysed. These same time periods were used for analysing the polysomnography data.

Blood pressure arousals. The raw PTT tracing was automatically processed by the RM50 software to remove artefact. The differences between adjacent PTT values were computed, and if they exceeded $50 \mathrm{~ms}$ (physiologically very unlikely), then the start of an artefact was marked. The software continued to record an artefact until three consecutive differences were $<50 \mathrm{~ms}$. Missing data were filled in by a linear interpolation for a maximum of 100 samples (20 s of data), after which a gap was left in the record. Data were next passed through a 17-sample (3.4 s) moving window average to remove most of the breath-bybreath oscillations. Finally, the data were processed for BP arousals (fall in PTT of $>15 \mathrm{~ms}$ lasting for $>5 \mathrm{~s}$ and $<45 \mathrm{~s}$ ) [19], and the number of arousals per hour of the PTT recording was calculated.

Inspiratory BP falls (inspiratory effort). The same artefact removal process was applied to the raw PTT data except that only five samples (1 s) of data separated by artefact were interpolated. Data were then passed through a three sample $(0.6 \mathrm{~s})$ moving window average. Finally, the tracing was processed for inspiratory BP falls that were defined as an inspiratory change (rise in PTT) lasting for $>0.7 \mathrm{~s}$ and $<4.5 \mathrm{~s}$. This definition was adapted from the work of DAVIES et al. [13]. The program calculated the number of breaths detected, and the mean and SD of the inspiratory PTT change (mean BP fall) for the whole PTT record.

Polysomnography data. Apnoea/hypopnoea index. Initial processing utilized the Oxford Medical 9200 replay system, which scored an apnoea when the amplitude of a breath (oronasal airflow) was reduced by $80 \%$ or more for at least $10 \mathrm{~s}$ and a hypopnoea when the breath amplitude was reduced by $50 \%$ for $10 \mathrm{~s}$ or more. The recording was later checked manually to exclude any apnoeas or hypopnoeas that had been scored during wakefulness. The number of apnoeas and hypopnoeas per hour were computed for the time period defined from the PTT record.

Oxygen saturation dip rate ( $\mathrm{Sa}, \mathrm{O}_{2}$ dips). Data processing utilized the Visi-Lab system (Stowood Scientific Instruments, Oxford, UK). The all-night $\mathrm{Sa}_{\mathrm{a}} \mathrm{O}_{2}$ data were stored every $12 \mathrm{~s}$, using the lowest $\mathrm{Sa}, \mathrm{O}_{2}$ value occurring in the previous $12 \mathrm{~s}$ period of the recording (this is identical to the data that would be stored and downloaded from the 
Ohmeda biox in trend output mode). The $12 \mathrm{~s}$ data were then used to calculate the number of $\mathrm{Sa}_{\mathrm{a}} \mathrm{O}_{2}$ dips of $>4 \%$ per hour of the analysis period.

EEG arousals. The Medilog Rapide software (Oxford Medical) was used to display the EEG, EOG's and EMG which were scored by one of the authors (D.J. Pitson) for arousals of $3 \mathrm{~s}$ or longer using the scoring rules published by the American Sleep Disorders Association (ASDA) [21]. The number of EEG micro-arousals per hour of the analysis period were computed.

All manual scoring was done blind to the other sleep study data and to the patient's clinical diagnosis.

Statistics. Examination of the data and the Shapiro-Wilk $\mathrm{W}$ test for non-normality suggested that most of the variables were unlikely to be normally distributed. Non-parametric methods were therefore used to examine the data. Arcus Pro-Stat (I.E. Buchan, West Lancashire, UK) was used for all statistics except for the receiver operator characteristics (ROC) curves which were obtained using True Epistat (Epistat Services, Richardson, TX, USA).

\section{Results}

The RM10 was tolerated well by the patients. PTT data from two laboratory studies and one home study could not be analysed because multiple ectopic beats clearly affected the PTT recording. The mean period $( \pm \mathrm{SD})$ of analysable PTT data was $6.5 \pm 1.64 \mathrm{~h}$ for the home studies and $6.1 \pm$ $2.22 \mathrm{~h}$ in the sleep laboratory.

\section{Reproducibility}

There was a reasonable agreement between the home and laboratory studies both for BP arousals (Spearman $\mathrm{r}=0.81$ ) and for inspiratory BP falls ( $\mathrm{r}=0.87)$. The Bland and Altman plots (fig. 1) suggest that subjects tended to have more $\mathrm{BP}$ arousals during the home study, mean difference (laboratory study - home study) $-3.9 \pm 10.9$ arousals per hour, and larger inspiratory BP falls, mean difference $-1.5 \pm 2.9 \mathrm{~ms}$.

\section{Clinical diagnosis}

According to the clinical diagnosis, 11 patients were found to be normal, 11 had a mild/moderate sleep disorder and 18 had a severe sleep disorder. Thirteen of the severe group were subsequently established on nasal CPAP. Of the remaining five, three patients tried CPAP but did not feel that the benefits outweighed the problems of this therapy, and two were unwilling to try CPAP and pursued alternative treatments.

\section{$B P$ arousals and inspiratory BP falls derived from PTT}

Figure 2 shows the data for BP arousals and inspiratory BP falls during the home study in the patients grouped according to their clinical diagnosis and in the group who were restudied having been established on nasal CPAP. BP arousals $\cdot \mathrm{h}^{-1}$ (median, 25-75\% range) were 10 (7-17),
21 (12-32), 36 (13-63), and 8 (7-13) for normal, mild/ moderate, severe and on CPAP groups, respectively. Inspiratory BP falls were 9 (8-12), 11 (9-15), 18 (13-21) and $8(6-10)$ for the same groups. Both BP arousals and inspiratory BP falls therefore show a clear progression from normal to severe that returns to the normal level on treatment. The Kruskal-Wallis test confirmed that there were significant differences between the clinical groups $(\mathrm{p}=0.0014$ for BP arousals, $\mathrm{p}<0.0001$ for inspiratory BP falls). The multiple comparisons procedure confirmed that for both $\mathrm{BP}$ arousals and inspiratory BP falls, there were no significant differences between the group classified as normal and the group restudied whilst on nasal CPAP, and between the normals and those with a mild/moderate disorder. Significant differences were found between the normal and severe group, the mild/moderate group and the group restudied on nasal CPAP, and the severe and on CPAP group. Inspiratory BP falls did differentiate between the mild/ moderate group and the severe group, but BP arousals did not (table 2).

Comparison between information derived from PTT and polysomnography data

Data from the laboratory sleep study enabled us to examine whether there were any differences between the ability
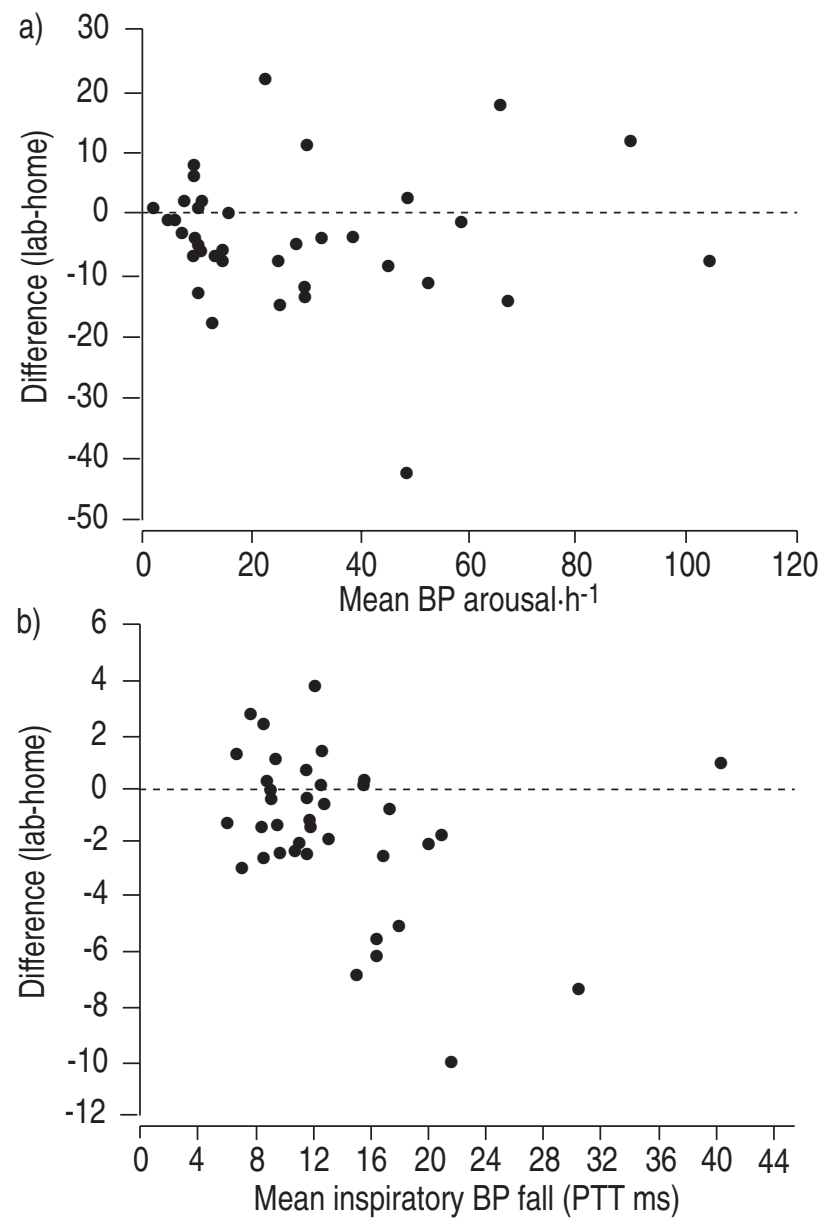

Fig. 1. - Agreement between the home and laboratory (lab) studies for the two derivatives of pulse transit time (PTT). a) Blood pressure (BP) arousals, and b) inspiratory BP falls. 

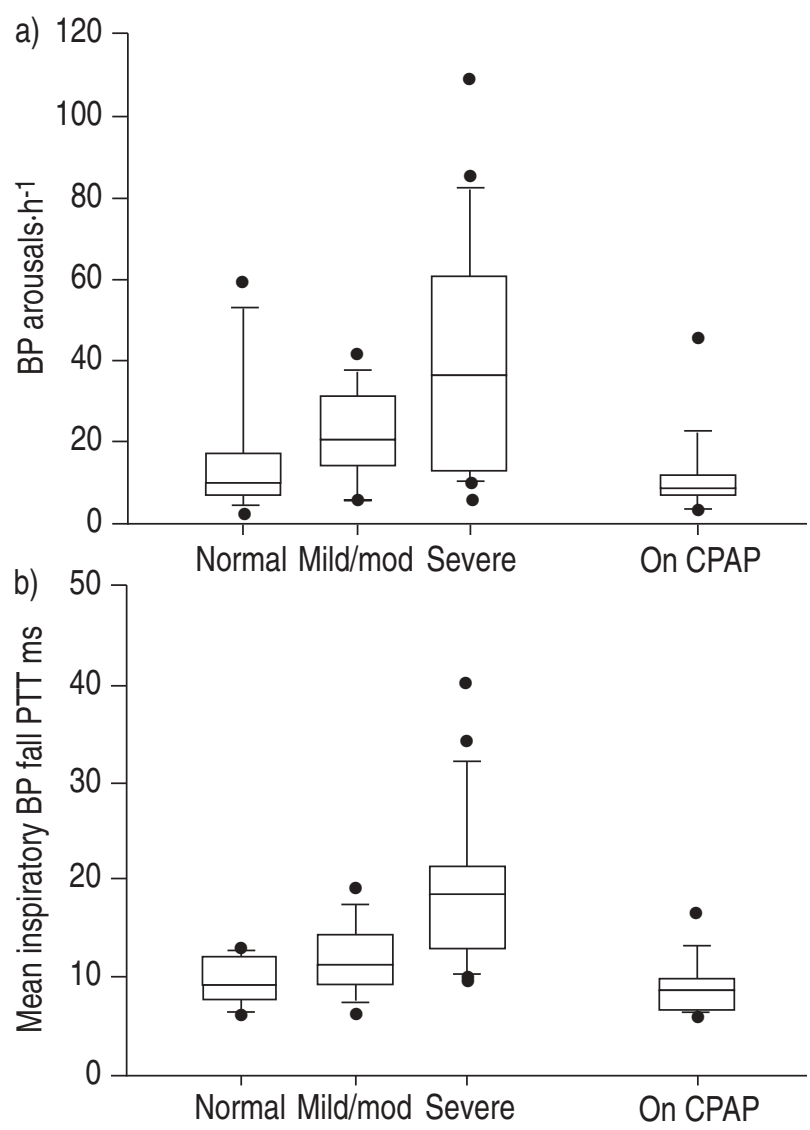

Fig. 2. - Box and whisker plots for the two pulse transit time (PTT) derivatives; a) blood pressure (BP) arousals, and b) inspiratory BP falls in patients grouped according to the severity of their sleep disorder and in 13 of the severe group who were restudied whilst using nasal continuous positive airway pressure (CPAP). The lower boundary of the box indicates the 25 th percentile, the line within the box indicates the median and the upper boundary of the box, the 75 th percentile. The 10th and 90th percentiles are indicated by the error bars below and above the box, and values outside the 10th and 90th percentile are shown as individual points.

of the PTT derived variables and other commonly measured sleep study variables (AHI, EEG micro-arousals, $\mathrm{Sa}_{\mathrm{a}} \mathrm{O}_{2}$ dips) to differentiate between the clinical groups. This was initially examined by the Kruskal-Wallis test. For all variables, significant differences were found between the groups, $\mathrm{S}_{\mathrm{a}} \mathrm{O}_{2}$ dips $\mathrm{p}<0.0001$, $\mathrm{AHI} \mathrm{p}=0.0002$, inspiratory $\mathrm{BP}$ falls $\mathrm{p}=0.0033$, EEG micro-arousals $\mathrm{p}=0.0037$, and $\mathrm{BP}$ arousals $p=0.025$. Table 3 shows the results of the multiple comparisons procedure that confirmed that all variables could discriminate between the normal and severe group, and all except BP arousals could discriminate between the

Table 2. - Kruskal-Wallis analysis for pulse transit time variables (home studies)

\begin{tabular}{lllllll}
\hline Clinical group & \multicolumn{2}{c}{ BP arousals $\cdot h^{-1}$} & \multicolumn{3}{c}{ Inspiratory BP falls } \\
\hline On CPAP & a & & & a & & \\
Normal & a & b & & a & b & \\
Mild/moderate & & b & c & & b & c \\
Severe disorder & & & c & & & c \\
\hline
\end{tabular}

$\overline{\text { Clinical groups with the same letter are not significantly differ- }}$ ent. Clinical groups with different letters are significantly different $(\mathrm{p}<0.05)$. BP: blood pressure; CPAP: continuous positive airway pressure.
Table 3. - Kruskal-Wallis analysis for all variables (laboratory studies)

\begin{tabular}{|c|c|c|c|c|c|c|c|}
\hline $\begin{array}{l}\text { Clinical } \\
\text { group }\end{array}$ & $\begin{array}{c}\text { Inspiratory } \\
\text { BP falls }\end{array}$ & $\begin{array}{l}\text { EEG micro- } \\
\text { arousals } \cdot \mathrm{h}^{-1}\end{array}$ & AHI & $\begin{array}{c}S_{\mathrm{a}, \mathrm{O}_{2}} \\
\text { dips } \cdot \mathrm{h}^{-1}\end{array}$ & \multicolumn{3}{|c|}{$\begin{array}{c}\text { BP } \\
\text { ousals } \cdot h^{-1}\end{array}$} \\
\hline Normal & $\mathrm{a}$ & $\mathrm{a}$ & $\mathrm{a}$ & $\mathrm{a}$ & $\mathrm{a}$ & & \\
\hline $\begin{array}{l}\text { Mild/ } \\
\text { moderate }\end{array}$ & $\mathrm{a}$ & $\mathrm{a}$ & $\mathrm{a}$ & $\mathrm{a}$ & a & & b \\
\hline $\begin{array}{l}\text { Severe } \\
\text { disorder }\end{array}$ & b & b & b & b & & & b \\
\hline
\end{tabular}

Clinical groups with the same letter are not significantly different. Clinical groups with different letters are significantly different $(p<0.05)$. BP: blood pressure; EEG: electroencephalogram; AHI: apnoea/hypopnoea index; $\mathrm{Sa}_{\mathrm{a}} \mathrm{O}_{2}$ : arterial oxygen saturation.

mild/moderate and severe group. None of the variables could discriminate between the normals and mild/moderate group.

A further comparison between the polysomnography and PTT variables was made by plotting ROC curves for each variable. In order to do this, the normal and mild/moderate groups were combined, resulting in two groups: patients offered a trial of nasal CPAP $(n=18)$ and patients not offered CPAP $(n=22)$. Analysis of the area under each ROC curve revealed that the respiratory variables $\left(\mathrm{AHI}\right.$ and $\mathrm{Sa}_{2} \mathrm{O}_{2}$ dips) had the highest areas under the curve followed by inspiratory BP falls, EEG micro-arousals and BP arousals. All variables were, however, highly statistically better than chance at differentiating between the two groups (fig. 3 ).

\section{Discussion}

The aim of this study was to examine whether inspiratory effort and BP arousals, measured indirectly from PTT, could contribute to the management of the OSAHS. The possible advantages of this technique are that it includes a noninvasive measure of both inspiratory effort and sleep fragmentation, using fully portable technology. In addition, since the measurement of PTT only requires ECG electrodes and an oximeter finger probe, and both of these are already used in other sleep monitoring systems, it is relatively simple to add PTT to existing systems. The equipment was easy to set up and use in the home, and patients tolerated it well. The data quality was good, with $93 \%$ of studies having analysable data. Our results confirmed that there was reasonable reproducibility of the two PTT derivatives over the two study nights, which was similar to that found by others [22]. When patients were subdivided according to the severity of their sleep disorder, both derivatives clearly reflected the increase in severity across the groups and showed a return to normal in patients who were restudied on treatment. When compared with some of the commonly measured polysomnography variables, the PTT-derived inspiratory effort was as good as EEG arousals, AHI and $\mathrm{Sa}, \mathrm{O}_{2}$ dips at discriminating between the three patient groups, and both PTT variables were comparable with the polysomnography variables when dividing the patients into the two management groups (offered a trial of nasal CPAP or not offered a trial of CPAP).

Only two patients' PTT data could not be analysed. In both cases, multiple ectopic beats, occurring at a frequency of between 7 and $10 \cdot \mathrm{min}^{-1}$, clearly affected the PTT. These resulted in sudden increases in PTT that either led to artefact or, if they were less than the $50 \mathrm{~ms}$ threshold 

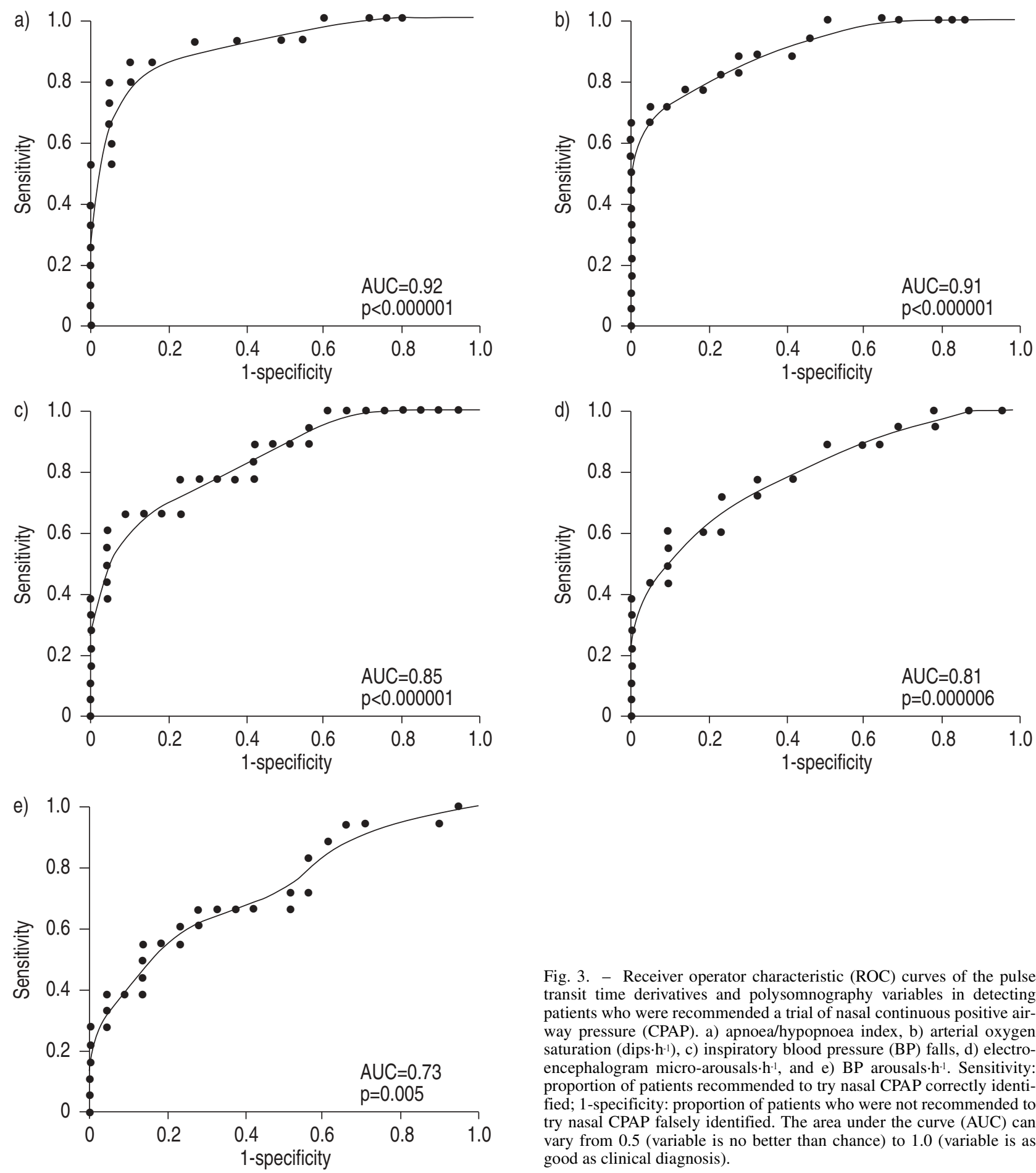

required for artefact rejection, a falsely long PTT. Because the ectopics were occurring within the respiratory frequency, this would have produced a high value for the average inspiratory BP fall if the studies had been analysed.

So far as the assessment of true reproducibility is concerned, this study is, of course, potentially flawed by the fact that the two recordings were undertaken under different environmental conditions. The first took place in the patient's home and the second in the sleep laboratory with the addition of further monitoring equipment. Both of the PTT derivatives suggested that the patient exhibited more

Fig. 3. - Receiver operator characteristic (ROC) curves of the pulse transit time derivatives and polysomnography variables in detecting patients who were recommended a trial of nasal continuous positive airway pressure (CPAP). a) apnoea/hypopnoea index, b) arterial oxygen saturation $\left(\operatorname{dips} \cdot \mathrm{h}^{-1}\right), \mathrm{c}$ ) inspiratory blood pressure (BP) falls, d) electroencephalogram micro-arousals $\cdot \mathrm{h}^{-1}$, and e) BP arousals $\cdot \mathrm{h}^{-1}$. Sensitivity: proportion of patients recommended to try nasal CPAP correctly identified; 1-specificity: proportion of patients who were not recommended to try nasal CPAP falsely identified. The area under the curve (AUC) can vary from 0.5 (variable is no better than chance) to 1.0 (variable is as good as clinical diagnosis).

abnormalities on the home study as the mean difference (laboratory study - home study) was negative for both. Agreement was better for inspiratory effort (mean difference $-1.5 \pm 2.9 \mathrm{~ms}$ ) than for BP arousals (mean difference $-3.9 \pm 10.9$ arousals $\cdot \mathrm{h}^{-1}$ ). A possible explanation for the higher number of arousals in the home study might be that the subjects slept longer than in the laboratory with less periods of wakefulness during the night. This agrees with the questionnaire that patients completed about their sleep. Patients were asked to rate how well they slept on each night compared with normal. Same as normal was scored 
as 1, a little less than normal as 2 and much less than normal as 3 . The mean scores for home and laboratory studies were $1.6 \pm 0.7$ and $2.1 \pm 0.7$, respectively, a difference that was significant $(\mathrm{p}<0.0001)$. The larger values for inspiratory effort would also suggest that sleep at home was more normal, allowing longer periods of obstructed breathing. Thus, we conclude that patients probably slept better with the portable equipment at home than they did during a polysomnography sleep study in the sleep laboratory, and that the results may therefore be more representative of their normal sleep.

The number of BP arousals per hour and the size of the inspiratory BP falls (degree of inspiratory effort ) monitored at home clearly increased with increasing disease severity (fig. 2). The one clear outlier in the normal group for BP arousals was a subject who was normal from the respiratory point of view but who had sleep disturbance from a cause that could not be identified. Both BP arousals and inspiratory BP falls also clearly documented the response to nasal CPAP treatment. The outlier who remains in the nasal CPAP group is a patient with severe obstructive sleep apnoea whose PTT derivatives suggest that their CPAP pressure is subtherapeutic. Despite this, the patient was considerably improved by CPAP (ESS before treatment 24 , on treatment 4) and was unwilling to tolerate a higher pressure as it led to no further reported improvement in well-being.

Analysis of the laboratory sleep study data revealed that the respiratory variables $\mathrm{AHI}$ and $\mathrm{Sa}_{\mathrm{a}} \mathrm{O}_{2}$ dips were the strongest discriminators between the normals and severe group and between the mild/moderate and severe group. None of the variables could distinguish between the normal and mild/moderate group. The results for the PTT variables were consistent with the home studies with both able to differentiate between normal and severe, but only inspiratory BP falls showing a separation between mild/moderate and severe. Once the patient group was divided into two, either offered a trial of nasal CPAP or not offered CPAP, all variables were significantly better than chance at detecting patients who clinically were felt to require a trial of nasal CPAP. Once again, the respiratory variables performed best, with areas under the curve $>0.90$. Inspiratory BP falls performed marginally better than EEG microarousals (area under the curve $=0.85$ compared with 0.81 ) with $\mathrm{BP}$ arousals performing least well (area under the curve $=0.73$ ).

The main limitation of any study looking at new diagnostic techniques in obstructive sleep apnoea is the absence of gold standard definitions of the disease. Original definitions based on apnoea, hypopnoeas, hypoxic dips or micro-arousals have not been shown to identify accurately those patients with a sleep fragmentation syndrome due to upper airway obstruction likely to respond to nasal CPAP. For example, Gulleminault et al. [23] identified patients with minimal apnoeas, hypopnoeas, hypoxic events and obvious arousals (upper-airway resistance syndrome) who clearly had hypersomnolence, increased upper-airway narrowing with inspiratory effort, and responded symptomatically to nasal CPAP. Thus, in our clinical grading of patients, there were some in the severe group who had relatively low $>4 \% \mathrm{Sa}_{\mathrm{a}} \mathrm{O}_{2}$ dip rates and $\mathrm{AHI}$ values, yet were very sleepy and responded well to nasal CPAP. Although PIETERS et al. [24] have found a weak correlation between CPAP use and a movement arousal index, in our unit at present, allocation of patients into the three categories is very much a clinical decision, based on history, degree of hypersomnolence and a sleep study designed to identify upper airway narrowing that leads to sleep fragmentation. This study was conceived to establish whether measurements of PTT-derived inspiratory effort and sleep fragmentation were as good as other, more conventional, indices at differentiating those put forward for a trial of nasal CPAP from those not. Clearly $>4 \% S \mathrm{a}, \mathrm{O}_{2}$ dip rate is likely to be very predictive, since this index was used as part of the diagnostic process, along with the other indices available from the Visi-Lab sleep study (heart rate oscillations, snoring and body movements), the ESS and the history. The PTT-derived variables were as good as the other polysomnography variables at dividing patients into these two groups according to nasal CPAP requirement, although when patients were divided into three groups, BP arousal numbers were unable to differentiate between the mild/ moderate and severe groups whereas all the other variables were.

If indirect BP monitoring was to form part of a portable sleep monitoring system, it would be unlikely that EEG would be recorded, and therefore no information about true sleep onset and sleep end would be available. For this reason, a "sensible" start and stop time for the analysis of the all night PTT data was established by excluding any obviously artefactual data at the start and end of the study if they existed and analysing the remaining data. In order to be able to compare the two PTT recordings (home and laboratory), the same approach was adopted for the laboratory recording, and this time period was applied to the analyses of EEG, AHI and $\mathrm{Sa}_{\mathrm{a}} \mathrm{O}_{2}$ dips.

In summary, recording overnight PTT in the home was easy to set up, well tolerated by the patients and produced reliable and reasonably reproducible results from its two derivatives, BP arousals and inspiratory BP falls (inspiratory effort). These two derivatives reflected the increase in disease severity across three clinically defined patient groups and the improvement on treatment and were similar to EEG micro-arousals, AHI and $\mathrm{Sa}_{\mathrm{a}} \mathrm{O}_{2}$ dips in their ability to differentiate between the patient groups.

We conclude that the measurement of indirect beat-tobeat blood pressure by using pulse transit time may be a useful addition to a portable monitoring system for the management of the obstructive sleep apnoea/hypopnoea syndrome.

\section{References}

1. Peter JH, Koehler U, Grote L, Podszus T. Manifestations and consequences of obstructive sleep apnoea. Eur Respir J 1995; 8: 1572-1583.

2. Bennett LS, Stradling JR. Who should receive treatment for sleep apnoea. Thorax 1997; 52: 103-104.

3. Gleeson K, Zwillich CW, White DP. The influence of increasing ventilatory effort on arousal from sleep. Am Rev Respir Dis 1990; 142: 295-300.

4. Gleeson K, Zwillich CW. Adenosine stimulation, ventilation, and arousal from sleep. Am Rev Respir Dis 1992; 145: 453-457.

5. Stradling JR. Sleep studies for sleep-related breathing disorders. A consensus report. J Sleep Res 1992; 1: $223-$ 230. 
6. Davies RJO, Stradling JR. The epidemiology of sleep apnoea. Thorax 1996; 51: Suppl 2, S65-S70.

7. Douglas NJ. Sleep-related breathing disorders: 3 - How to reach a diagnosis in patients who may have the sleep apnoea/hypopnoea syndrome. Thorax 1995; 50: 883-886.

8. Hilton SM. The defence-arousal system and its relevance for circulatory and respiratory control. J Exp Biol 1982; 100: 159-174.

9. Martin SE, Dreary IJ, Douglas NJ. The effect of autonomic arousals on daytime function. [Abstract]. Am J Respir Crit Care Med 1996; 153(2): 354.

10. Martin SE, Wraith PK, Deary IJ, Douglas NJ. The effect of nonvisible sleep fragmentation on daytime function. Am J Respir Crit Care Med 1997; 155: 1596-1601.

11. Lea S, Ali NJ, Goldman M, et al. Systolic blood pressure swings reflect inspiratory effort during simulated obstructive sleep apnoea. In: Horne J, ed. Sleep 90. Bochum, Pontenagel Press, 1990; pp. 178-181.

12. Davies RJO, Belt PJ, Robert SJ, Ali NJ, Stradling JR. Arterial blood pressure responses to graded transient arousal from sleep in normal humans. J Appl Physiol 1993; 74: 1123-1130.

13. Davies RJO, Vardi-Visy K, Clarke M, Stradling JR. Identification of sleep disruption and sleep disordered breathing from the systolic blood pressure profile. Thorax 1993; 48: 1242-1247.

14. Gribbin B, Steptoe A, Sleight P. Pulse wave velocity as a measure of blood pressure change. Psychophysiology 1976; 13: 86-90.

15. Pitson DJ, Chhina N, Knijn S, van Herwaaden M, Stradling JR. Mechanism of pulse transit time lengthening during obstructed inspiration. J Amb Mon 1995; 8: 101105.
16. Brock J, Pitson D, Stradling JR. Use of pulse transit time as a measure of changes in inspiratory effort. J Amb Mon 1993; 6: 295-302.

17. Pitson DJ, Sandell A, van den Hout R, Stradling JR. Use of pulse transit time as a measure of inspiratory effort in patients with obstructive sleep apnoea. Eur Respir J 1995; 8: 1669-1674.

18. Pitson D, Chhina N, Knijn S, van Herwaaden M, Stradling J. Changes in pulse transit time and pulse rate as markers of arousal from sleep in normal subjects. Clin Sci Colch 1994; 87: 269-273.

19. Pitson DJ, Stradling JR. Autonomic markers of arousal during sleep in patients undergoing investigation for obstructive sleep apnoea, their relationship to EEG arousals, respiratory events and subjective sleepiness. J Sleep Res 1998; 7: 53-59.

20. Johns MW. A new method for measuring daytime sleepiness: the Epworth sleepiness scale. Sleep 1991; 14: 540545.

21. Bonnet M, Carley D, Carskadon M, et al. EEG arousals: scoring rules and examples. A preliminary report from the Sleep Disorders Atlas Task Force of the American Sleep Disorders Association. Sleep 1992; 15(2): 173-184.

22. Flemons WW, Remmers JE. The diagnosis of sleep apnea: questionnaires and home studies. Sleep 1996; 19: S243-S247.

23. Guilleminault C, Stoohs R, Clerk A, Cetel M, Maistros P. A cause of excessive daytime sleepiness. The upper airway resistance syndrome. Chest 1993; 104: 781-787.

24. Pieters T, Collard P, Aubert G, Dury M, Delguste P, Rodenstein DO. Acceptance and long-term compliance with nCPAP in patients with obstructive sleep apnoea syndrome. Eur Respir J 1996; 9: 939-944. 